\title{
Application of spray granulation for conversion of mixed phospholipid-bile salt micelles to dry powder form: influence of drug hydrophobicity on nanoparticle reagglomeration
}

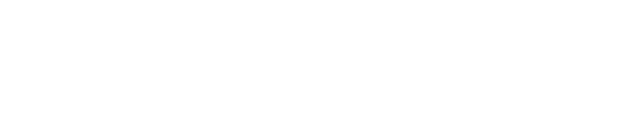

\author{
Qingyuan Lv' \\ Xianyi $\mathrm{Li}^{2}$ \\ Baode Shen' \\ $\mathrm{He} \mathrm{Xu}^{\prime}$ \\ Chengying Shen' \\ Ling Dai' \\ Jinxia Bai' \\ Hailong Yuan' \\ Jin Han'
}

'Department of Pharmacy, 302 Military Hospital, ${ }^{2}$ Institute for Drug and Instrument Control, Health Department, General Logistics Department of People's Liberation Army, Beijing, People's Republic of China
Correspondence: Hailong Yuan; Jin Han Department of Pharmacy, 302 Military Hospital, Beijing, People's Republic of China Tel +86 I0 66933367 Fax +86 I0 63879589 Email yhlpharm@।26.com; hanjinemba@।63.com

\begin{abstract}
The aim of this study was to investigate the feasibility of using spray granulation as a drying method to convert phospholipid (PL)-sodium deoxycholate (SDC)-mixed micelles (MMs) containing a water-insoluble drug to a solid dosage form and to evaluate how drugs with significantly different physicochemical properties affect the spray granulation process and subsequent in vitro and in vivo processes. Cucurbitacin $\mathrm{B}(\mathrm{Cu} \mathrm{B})$ and glycyrrhizin (GL) were used as the model drugs. After spray granulation, the dried Cu B-PL/SDC-MM powder was completely redispersible within 15 minutes in vitro. Meanwhile, the area under the curve during 24 hours $\left(\mathrm{AUC}_{0-24}\right)$ and peak serum concentration from the dried powder were significantly $(P<0.05)$ lower than the values from $\mathrm{Cu}$ B-PL/SDC-MMs in vivo. However, a better result was obtained for GL, ie, the drug was redispersed completely within 5 minutes in vitro. Further, absorption from the dried GL-PL/SDC-MM powder was increased to the same level as that for GL-PL/SDC-MMs in vivo compared with the control group. The difference in these results can be found in $\mathrm{Cu} \mathrm{B}$ and GL. $\mathrm{Cu} \mathrm{B}$ nanoparticles reagglomerated when released, resulting in slower redispersibility and less absorption compared with the original PL-SDC-MMs. However, no agglomeration or delay was observed for GL. A possible explanation is the difference in surface hydrophobicity between $\mathrm{Cu} \mathrm{B}$ and GL. The results of this study not only show that spray granulation is an effective drying technique that can complement spray-drying and freeze-drying, but also confirm that the physicochemical properties of a drug have a significant influence on the in vitro and in vivo performance of the dried powder obtained after spray granulation.
\end{abstract}

Keywords: cucurbitacin B, glycyrrhizin, mixed phospholipid-bile salt micelles, fluid bed granulation, poorly water soluble drugs

\section{Introduction}

Oral administration is the preferable route for patients, owing to its safety and compliance. In 2012, sales for oral drugs in the US alone were estimated to be $\$ 56.7$ billion. ${ }^{1}$ Thus, the development of novel drugs and technologies which are effective via oral administration is an important task for pharmaceutical researchers. However, in recent decades, new active pharmaceutical ingredients tend to have a higher molecular mass and a higher degree of hydrophobicity, resulting in them being less soluble in water. ${ }^{2}$ Up to $40 \%$ of newly discovered active pharmaceutical ingredients have a solubility problem. ${ }^{3}$ Oral delivery of these drugs invariably suffers from erratic absorption, low bioavailability, high intrasubject and intersubject variation, and lack of dose proportionality. ${ }^{4}$ Therefore, it is a genuine challenge to deliver water-insoluble drugs via the oral route. 
Several approaches have been taken to solve this problem, including self-microemulsifying formulations, salt formulations, solid dispersions, and micronization/nanonization. Among these applications, use of nanoparticles prepared as mixed phospholipid-bile salt micelles (PL-BS-MMs) as a way to formulate poorly soluble drugs has matured rapidly in recent years due to their physiological compatibility and solubilizing capacity. ${ }^{5,6}$ Further, in order to benefit from the advantages related to a solid dosage form and to obtain a long-term stable formulation, it is often necessary to convert liquid PL-BS-MMs to a dry powder form which can be subsequently compressed into a tablet or encapsulated from the perspective of physical stability and patient compliance. During the solidification process, solid nanoparticles can be formed by evaporation of the solvent from the mixed micelle solution. Solid formulation of PL-BS-MMs represents a novel drug delivery system for improving the physical and chemical stability of PL-BS-MMs, and is composed of a drug with stabilizers or protectants, and can be reconstituted instantaneously on mild agitation or peristalsis followed by dilution with aqueous medium in vitro or in the gastrointestinal tract. ${ }^{7}$ Because aggregation is particularly prone to occurring during the drying process, and usually results in an inability to retain the desired nanometer particle size, the art of solidification technology has become particularly important. Two major solidification methods for this transformation include freeze-drying and spraydrying. ${ }^{78}$ However, powders yielded by either of these two processes tend to have poor properties, such as high bulk density, high hygroscopicity, or poor fluidity. Further, in order to improve the processability further, extra ingredients need to be added to the powders which are obtained from spray-drying or freeze-drying. The spray granulation-based approach would be an alternative solution to the above problems. However, after reviewing the current literature, there are very few reports referring to application of the spray granulation-based approach for converting PL-BS-MMs to dry powder form. ${ }^{9}$ Reports focusing on the process of spray granulation and the influence of drug properties and type of protectants or stabilizers used are even scarcer.

In order to investigate the influence of drug properties, two model drugs, ie, cucurbitacin B (Cu B) and glycyrrhizin (GL), were chosen based on their distinct difference in surface hydrophobicity, with GL having the lower hydrophobicity. ${ }^{10,11} \mathrm{Cu} \mathrm{B}$, the major compound in cucurbitacin, has a broad range of biological activity, including anti-inflammatory, hepatocurative, and hepatoprotective effects. ${ }^{12-14} \mathrm{GL}$ is one of the main components extracted from
Glycyrrhiza glabra L. and it possesses a variety of pharmacological effects, including anti-inflammatory, antiviral, and antioxidative activity. ${ }^{15-17}$ Both have potent pharmacological activity, but suffer from low bioavailability following oral administration due to poor water solubility.

Therefore, the aim of the present study was three-fold. First, the effect of concentration and type of stabilizer used in the solidification process was investigated. Second, the influence of surface hydrophobicity of the drug on redispersibility was studied in vitro. Third, in order to determine whether the granulated powders maintained good redispersibility in vivo, a comparative bioavailability study of the different formulations was performed in fasted male Beagle dogs.

\section{Materials and methods \\ Materials}

Cucurbitacin B (98\%) was sourced from Nantong Feiyu Biological Technology Co, Ltd (Nantong, People's Republic of China); GL (98\%) from Sigma Chemical Company (St Louis, MO, USA); phospholipid (PL, >95\%) from Shanghai TaiWei Pharmaceutical Industry Co, Ltd (Shanghai, People's Republic of China); sodium deoxycholate (SDC) from Beijing Hotaibio Science and Technology Co, Ltd (Beijing, People's Republic of China); hydroxypropyl methyl cellulose (HPMC) $5 \mathrm{mPa} \cdot \mathrm{s}$ from Colorcon, Inc (Shanghai, People's Republic of China); alpha-D-tocopherol polyethylene glycol 1000 succinate (TPGS) from ISOCHEM SAS (Gennevilliers, France); mannitol DC from Roquette (Lestrem, France); and methanol of high performance liquid chromatography (HPLC) grade from Promptar Co, Ltd (Elk Grove, CA, USA). Other reagents and solvents were of analytical or HPLC grade.

\section{Preparation and characterization of PL-SDC-MMs}

\section{Preparation}

Preparation of drug loaded phospholipid (PL)-sodium deoxycholate (SDC)-mixed micelles (MMs) was carried out using a film dispersion method based on our previous research. ${ }^{10,11}$ In the optimized formulation, the total concentration of $\mathrm{PL} / \mathrm{SDC}$ was $54 \mathrm{mg} / \mathrm{mL}$ and the mass concentration ratio was 1:0.8. Briefly, PL and SDC were dissolved by anhydrous ethanol in a round-bottom flask. A thin film was formed after evaporation of the organic solvent using a rotary evaporator (Laborota 4000, Heidolph Instruments $\mathrm{GmbH} \& \mathrm{Co}, \mathrm{KG}$, Germany). The film was rehydrated in a given amount of distilled water to give a clear solution. $\mathrm{Cu} \mathrm{B}$ (mass concentration $5 \mathrm{mg} / \mathrm{mL}$ ) and GL (mass concentration $40 \mathrm{mg} / \mathrm{mL}$ ) 
were added to this solution, then nitrogen-sealed and mixed for 24 hours on a magnetic stirrer (85-2, Guohua Instrument Co, Ltd, Changzhou, People's Republic of China) at room temperature to form clarified PL-SDC-MMs.

\section{Particle size and zeta potential determination}

The particle size and polydispersity index of the $\mathrm{Cu}$ B-PL/SDC-MMs and GL-PL/SDC-MMs were determined by photon correlation spectroscopy and a Zetasizer $(3000 \mathrm{SH}$, Malvern Instruments, Malvern, UK), respectively. Prior to detection, the samples were diluted with double-distilled water to achieve the required dilution for photon correlation spectroscopy. Each sample was measured at a fixed angle of $90^{\circ}$ at $25^{\circ} \mathrm{C}$ in triplicate. The zeta potential values were assessed by determining the particle electrophoretic velocity using the same instrument.

\section{Studies of physical stability}

The $\mathrm{Cu}$ B-PL/SDC-MM and GL-PL/SDC-MM solutions were both stored at room temperature. Particle size, zeta potential, and polydispersity index were determined after storage for $10,20,30,60$, and 90 days to evaluate their physical stability using the same method as described for determination of particle size and zeta potential.

\section{Preparation and characterization of granulated powder} Spray granulation of PL-SDC-MMs

Spray granulation of the PL-SDC-MMs was performed using a mini fluid bed dryer (Glatt Air Techniques, Inc, Ramsey, NJ, USA). The parameters used in the granulation process were as follows: the mode was top spray, the feed rate was $3 \mathrm{~g}$ per minute, the atomizing air pressure was 2.0 bars, the inlet air temperature was $50^{\circ} \mathrm{C}$, and the substrate on which the PL-SDC-MMs were sprayed was mannitol DC. The temperature for the product was kept constant in the range of $35^{\circ} \mathrm{C}-40^{\circ} \mathrm{C}$ throughout the process. These parameters were set on the basis of preliminary experiments and kept constant for all the following experiments. HPMC and TPGS were selected as stabilizers. The composition or identity of the PL-SDC-MMs was coded according to the stabilizer and drug used in formulation; for example, GL-TPGS10 indicates a PL-SDC-MMs containing TPGS as the stabilizer in a mass concentration of $10 \mathrm{mg} / \mathrm{mL}$ and GL as the drug. The size of each batch was $60 \mathrm{~g}$ and the theoretical drug load was $20 \%$ (12 g) for $\mathrm{Cu} \mathrm{B}$ and GL with respect to the substrate, respectively. Before starting spray granulation, the fluid bed dryer should be preheated. In order to avoid agglomeration or clumping of the powder, sufficient fluidization of the substrate should also be maintained during the granulation process. At the end of the spray granulation process, the powders were cooled down to room temperature, screened by a 20 mesh screen, and stored in a refrigerator (at $2^{\circ} \mathrm{C}-8^{\circ} \mathrm{C}$ ) until further analysis.

\section{Particle size analysis of powder obtained after granulation}

Particle size was measured using a laser particle size analyzer (Winner 801; Jinan Winner Particle Instrument Stock Co, Ltd, Jinan, People's Republic of China) to investigate the likelihood of reagglomeration of the nanoparticles after their release. Prior to measurement, the granulated powders were milled using a CryoMill (Retsch GmbH, Haan, Germany) at $20 \mathrm{~Hz}$ for 1.5 minutes. The powders were then diluted with distilled water to achieve a concentration suitable for analysis. For each sample, measurements were performed with and without ultrasonication. The same parameters were set for the PL-SDC-MMs.

\section{Determination of drug loading in dried PL-SDC-MMs}

The drug loaded into the dried PL-SDC-MMs was detected using the HPLC method. Briefly, a known amount of powder was dissolved in methanol, sonicated for 20 minutes, and then centrifuged for 10 minutes $(4,000 \mathrm{rpm})$. The supernatants were collected and then diluted with methanol before analysis. Chromatographic separation was performed using a Pinnacle DB C18 column $(250 \times 4.5 \mathrm{~mm}, 5 \mu \mathrm{m}$, Agilent Technologies, Palo Alto, CA, USA) at $25^{\circ} \mathrm{C}$, protected using an Alltima ${ }^{\mathrm{TM}}$ (Alltech Associates Inc., Deerfield, IL, USA) C18 guard column $(20 \times 4 \mathrm{~mm}, 5 \mu \mathrm{m})$. The DAD detector was set at $228 \mathrm{~nm}$ and $254 \mathrm{~nm}$ for $\mathrm{Cu} \mathrm{B}$ and GL, respectively. The mobile phase for $\mathrm{Cu} B$ was $68 \%$ (v/v) of methanol and $32 \%(\mathrm{v} / \mathrm{v})$ of deionized water solution, and for GL was $60 \%$ $(\mathrm{v} / \mathrm{v})$ of methanol and $40 \%(\mathrm{v} / \mathrm{v})$ of ammonium acetate $(0.2$ $\mathrm{mol} / \mathrm{L})$. A flow rate of $1.0 \mathrm{~mL}$ per minute and $20 \mu \mathrm{L}$ of injection volume were used for both active pharmaceutical ingredients.

\section{Redispersibility test}

Given that the granulated powders can reconstitute and form mixed micelles in water, the term "redispersibility" is preferable to "dissolution". "The redispersibility of the formulations and crude drugs was studied in $500 \mathrm{~mL}$ of different types of medium using USP apparatus 1 at $100 \mathrm{rpm}$ and $37^{\circ} \mathrm{C}(\mathrm{ZRS}-8 \mathrm{G}$ dissolution tester; Tianda Tianfa Technology Co, Ltd, Tianjin, People's Republic of China) for Cu B and GL. The medium was 
demineralized water composed of $0.4 \%$ sodium lauryl sulfate for GL and demineralized water composed of 1.5\% sodium lauryl sulfate for $\mathrm{Cu}$ B. Samples were withdrawn at predetermined time intervals. Rapid stirring (200 rpm) was performed for 45-60 minutes. Concentrations of the samples taken were determined by HPLC which consisted of a quaternary pump (G1100A Quat Pump), degasser, diode array detector (G1100A DAD), and HP ChemStation Data system (Agilent Technologies). Separations were performed on a Pinnacle DB C18 column $(250 \times 4.5 \mathrm{~mm}, 5 \mu \mathrm{m}$, Agilent Technologies $)$ at $25^{\circ} \mathrm{C}$, protected by an Alltima C18 guard column $(20 \times 4 \mathrm{~mm}$, $5 \mu \mathrm{m})$. The DAD detector was set at $228 \mathrm{~nm}$ and $254 \mathrm{~nm}$ for $\mathrm{Cu} \mathrm{B}$ and GL, respectively. The mobile phase for $\mathrm{Cu} \mathrm{B}$ was $68 \%(\mathrm{v} / \mathrm{v})$ of methanol and $32 \%(\mathrm{v} / \mathrm{v})$ of deionized water solution, and for GL was $60 \%(\mathrm{v} / \mathrm{v})$ of methanol and $40 \%(\mathrm{v} / \mathrm{v})$ of ammonium acetate $(0.2 \mathrm{~mol} / \mathrm{L})$. A flow rate of $1.0 \mathrm{~mL}$ per minute and an injection volume of $20 \mu \mathrm{L}$ were used for both active pharmaceutical ingredients. Every experiment was performed six times.

\section{Surface morphology was determined by scanning electron microscopy}

The morphology of granulated powder samples was observed using a scanning electron microscope (S-4800; Hitachi Technologies Corporation, Tokyo, Japan). The samples were coated with gold and palladium using a vacuum evaporator and examined at an accelerating voltage of $15 \mathrm{kV}$.

\section{Bioavailability study}

The protocol for our study was reviewed and approved by the 302 Military Hospital of China Institutional Animal Care and Use Committee. The drugs were formulated and prepared as PL-SDC-MMs and as PL-SDC-MM spray granulation powders. Coarse $\mathrm{Cu}$ B and coarse GL were used as controls. The powders were filled into capsules. Different formulations were administered orally to fasted male Beagle dogs $(n=5$, body weight $10-11 \mathrm{~kg}$ ) in a crossover study at a dose of $1 \mathrm{mg} / \mathrm{kg}$ for $\mathrm{Cu} \mathrm{B}$ and $100 \mathrm{mg} / \mathrm{kg}$ for GL. ${ }^{10,11}$ The washout period between dosing with each formulation was 7 days. A blood sample of approximately $2 \mathrm{~mL}$ was collected from each animal at 0 (pre-dose), and $0.25,0.5,1,1.5,2,3,4,6,8,10,12$ and 24 hours post-dose. The collected blood samples were placed in heparinized tubes and separated immediately by centrifugation (H 2050R; Xiang Yi Centrifuge Instrument Co, Ltd, Hunan, People's Republic of China) at 8,000 rpm for 10 minutes at $4^{\circ} \mathrm{C}$ to yield plasma and stored at $-40^{\circ} \mathrm{C}$ prior to analysis.

The $\mathrm{Cu} \mathrm{B}$ concentration in the biological samples was assayed by the HPLC method described previously. Briefly, the mobile phase was methanol- $\mathrm{H}_{2} \mathrm{O}(64: 36, \mathrm{v} / \mathrm{v})$, and the flow rate and ultraviolet wavelength were $1.0 \mathrm{~mL}$ per minute and $228 \mathrm{~nm}$, respectively. $\mathrm{Cu} \mathrm{B}$ and the internal standard methyl-norethindrone were extracted from plasma using a simple protein precipitation procedure with four-fold acetonitrile, followed by centrifugation at 10,000 rpm for 20 minutes. The organic phase was collected and dried by blowing nitrogen at $30^{\circ} \mathrm{C}$. The dried residue was dissolved in $0.1 \mathrm{~mL}$ of methanol and injected into the HPLC column.

The deproteinization method was used to prepare the GL plasma samples as follows. First, $400 \mu \mathrm{L}$ methanol was added to each $200 \mu \mathrm{L}$ plasma sample. The mixtures were then whirled (MS3; IKA ${ }^{\circledR}$, Staufen, Germany) for 2 minutes and centrifuged at 3,000 rpm for 5 minutes. Thereafter, a $20 \mu \mathrm{L}$ volume of the obtained supernatant was directly injected into the HPLC system for analysis. Because most GL transits through the small intestine and is hydrolyzed to glycyrrhetinic acid (the active metabolite) by the action of intestinal bacteria in the large intestine, the resulting glycyrrhetinic acid is absorbed into the systemic circulation. Thus, glycyrrhetinic acid is detectable at a significant concentration in plasma after oral administration of GL. The HPLC system consisted of a quaternary pump (G1100A QuatPump), a degasser, a diode array detector (G1100A DAD), and a HP ChemStation Data system (all from Agilent Technologies). The analysis was performed on a Pinnacle DB C18 column $(250 \times 4.5 \mathrm{~mm}$, $5 \mu \mathrm{m}$, Agilent Technologies) protected by an Alltima C18 guard column $(20 \times 4 \mathrm{~mm}, 5 \mu \mathrm{m})$. The DAD detector was set at a wavelength of $250 \mathrm{~nm}$. The column temperature was maintained at $25^{\circ} \mathrm{C}$. The mobile phase was a mixture of methanol/ammonium acetate $(0.2 \mathrm{~mol} / \mathrm{L}), 65: 35, \mathrm{v} / \mathrm{v}$, pumped at a flow rate of $1 \mathrm{~mL}$ per minute.

The pharmacokinetic analysis was performed using DAS $^{\circledR} 2.0$ (Boying Corporation, Beijing, People's Republic of China). The data were subjected to a one-way analysis of variance followed by the least significant difference post hoc test. The results were presented as the mean and standard error of mean.

\section{Result and discussion Preparation and characterization of PL-SDC-MMs}

The active pharmaceutical ingredient was embedded into the hydrophobic core of the mixed micelles under mechanical force, isolating it from the medium. Based on our previous studies, the two PL-SDC-MMs were both spherical in shape with particle sizes around $100 \mathrm{~nm}$ and a narrow distribution ( $90.31 \pm 8.32 \mathrm{~nm}$ and polydispersity index 0.146 , respectively, 
for $\mathrm{Cu} \mathrm{B}-\mathrm{PL} / \mathrm{SDC}-\mathrm{MMs} ; 82.99 \pm 7.5 \mathrm{~nm}$ and polydispersity index 0.096 for GL-PL/SDC-MMs). The zeta potential is an important factor in evaluating the stability of a colloidal dispersion. ${ }^{18}$ Generally speaking, particles are stable when the absolute value of the zeta potential is above $30 \mathrm{mV}$ due to electric repulsion between particles. ${ }^{19-21}$ The zeta potential of the $\mathrm{Cu}$ B-PL/SDC-MMs and GL-PL/SDC-MMs was -31.21 11.17 $\mathrm{mV}$ and $-32.23 \pm 1.05 \mathrm{mV}$, respectively, suggesting that both of the mixed micelle liquids were dynamic stable systems. The concentration of $\mathrm{Cu} \mathrm{B}$ and GL in our optimized PL-SDCMMs system was $5 \mathrm{mg} / \mathrm{mL}$ and $40 \mathrm{mg} / \mathrm{mL}$, respectively. For $\mathrm{Cu} \mathrm{B}$, more PL/SDC was needed to obtain a PL-SDC-MM system due to its more hydrophobic surface. The optimized $\mathrm{Cu}$ B-PL/SDC-MMs and GL-PL/SDC-MMs remained stable colloidal systems for 90 days with a constant particle size and encapsulation efficiency.

\section{Preparation and characterization of granulated powders}

Formulation developed

The same parameters were used to granulate the PL-SDCMMs and investigate whether they could be granulated as such. All samples investigated and the results for coating efficiency are listed in Table 1 . The coating efficiency was calculated as follows:

$$
\text { Coating efficiency }=\frac{\text { Drug load }}{\text { Theoretical drug load }} \times 100 \% \text {. }
$$

Due to the amount of stabilizer being different in the different formulations, it would have been difficult to compare the differences between formulations by calculating the drug load with respect to the whole formulation. Therefore, in this study, the drug load in the granules was calculated with

Table I Results of coating efficiency for different formulations (mean \pm standard deviation, $\mathrm{n}=6$ )

\begin{tabular}{ll}
\hline Code & Coating efficiency (\%) \\
\hline GL-HPMC20 & $76.37 \pm 3.11$ \\
GL-HPMC30 & $88.64 \pm 4.17$ \\
GL-HPMC50 & $94.09 \pm 2.88$ \\
GL-TPGS10 & $63.11 \pm 2.39$ \\
GL-TPGS20 & $81.47 \pm 3.29$ \\
Cu B-HPMC20 & $69.23 \pm 2.77$ \\
Cu B-HPMC30 & $89.21 \pm 3.30$ \\
Cu B-HPMC50 & $94.28 \pm 2.04$ \\
Cu B-TPGSI0 & $59.22 \pm 3.44$ \\
Cu B-TPGS20 & $69.36 \pm 3.06$ \\
\hline
\end{tabular}

Abbreviations: GL, glycyrrhizin; HPMC, hydroxypropyl methyl cellulose; Cu B, Cucurbitacin B; TPGS, alpha-D-tocopherol polyethylene glycol 1000 succinate. respect to the substrate rather than the whole formulation. In the process of granulation, the PL-SDC-MMs were atomized to produce quality droplets at a high pressure of 2.0 bars. Under this high pressure, droplets could be spread uniformly over the substrates. Meanwhile, these very fine droplets could lead to the minimum localized wetting to the substrates. As we know the importance of retaining the fast redispersion profile of the solid PL-BS-MMs, the formulation with poor granule strength, which could lead to fast re-dispersion profile, was highly desirable. ${ }^{22}$ Therefore, in order to reduce the availability of the binder to form liquid bridges between particles, a low PL-SDC-MMs feed rate of 3 g per minute was set. The fewer the liquid bridges in a formulation, the more friable the granules are. ${ }^{23}$ Generally speaking, there are three processes involved in spray granulation, ie, wetting and nucleation, consolidation and then final breakage and attrition. All these processes are governed by the physicochemical properties of the stabilizer, the properties of substrate, and the parameters set during preparation. During this spray granulation experiment, the substrate used in the formulations was the same (mannitol DC), and constant processing parameters were kept for all batches. Therefore, the function of the concentration and type of stabilizer were the main factors contributing to the properties of the granules.

As shown in Table 1, the more stabilizer used in the formulation, the higher the drug load detected in the granules. This indicates that the amount of stabilizer plays an important role in making a drug adhere to a substrate. Actually, the TPGS and HPMC had a dual function in the drying process, ie, acting as binders to adhere the nanoparticles to their substrate and preventing agglomeration of the nanoparticles. The granulation process is regarded as successful if the coating efficiency is $90 \%$ or greater. ${ }^{24}$ Based on this standard, formulations with a concentration of $30 \mathrm{mg} / \mathrm{mL}$ or $50 \mathrm{mg} / \mathrm{mL}$ of HPMC were used for further study of both drugs without further treatment. After considering the standard deviation, the coating efficiency of formulations with a concentration of $30 \mathrm{mg} / \mathrm{mL}$ HPMC was also within the acceptable range. However, we chose only those formulations with a concentration of $50 \mathrm{mg} / \mathrm{mL}$ for the subsequent redispersibility experiments. Formulations with a different concentration of TPGS did not reach sufficient coating efficiency. However, in order to demonstrate the wide application of spray granulation technology and to further widen the scope of this study, we decided to do further work on the formulation with TPGS as the stabilizer for PL-SDC-MMs, in the hope of providing more information on the critical parameters of the spray granulation by further optimization. 


\section{Optimizing formulations with TPGS to increase coating efficiency}

According to the previous data, there are two solutions which could be used to increase the coating efficiency of the formulation with TPGS. Based on the results shown in Table 1, our first thought was to increase the amount of TPGS due to the upward trend detected in the previous study. However, excessive use of TPGS could cause side effects because TPGS has biological activity in itself. As we know, HPMC has excellent film-forming properties, and good results were obtained in the earlier part of the study. Thus, another choice was to add extra HPMC to the formulation with TPGS to improve the coating efficiency.

For GL-TPGS10, more than $90 \%$ coating efficiency was obtained when the concentration of HPMC reached $30 \mathrm{mg} / \mathrm{mL}$ (Table 2). However, sufficient coating efficiency could not be reached for Cu B-TPGS10, although the concentration of HPMC was $30 \mathrm{mg} / \mathrm{mL}$. On the other hand, for Cu B-TPGS20, the formulation could be coated easily when a concentration of $30 \mathrm{mg} / \mathrm{mL}$ was used for HPMC.

The surface morphology of the granulated powders was visualized using scanning electron microscopy (Figure 1) in order to find out why the coating efficiency was low. Figure $1 \mathrm{~A}$ and $\mathrm{B}$ show the granulated powders containing $\mathrm{Cu}$ B-TPGS20-HPMC30 and GL-HPMC50, respectively. The granulated powders containing Cu B-TPGS10-HPMC30 are shown in Figure 1C. It is easy to distinguish the quality of these products from these figures. The granulated powders containing Cu B-TPGS20-HPMC30 and GL-HPMC50 have a much smoother surface than those containing Cu B-TPGS10HPMC30. The defects on the surface of these granulated powders can be clearly observed in Figure 1C. A possible explanation for these defects is failure of the spray granulation process due to the mixed micelle solution not adhering well to the substrate, and this would account for the low coating efficiency.

Table 2 Results of coating efficiency for formulations containing additional HPMC (mean \pm standard deviation, $n=6$ )

\begin{tabular}{ll}
\hline Code & Coating efficiency (\%) \\
\hline GL-TPGSI0-HPMC20 & $83.33 \pm 3.17$ \\
GL-TPGSI0-HPMC30 & $92.37 \pm 2.46$ \\
Cu B-TPGSI0-HPMC20 & $73.88 \pm 3.08$ \\
Cu B-TPGSI0-HPMC30 & $85.24 \pm 3.1$ I \\
Cu B-TPGS20-HPMC20 & $82.79 \pm 3.71$ \\
Cu B-TPGS20-HPMC30 & $94.33 \pm 2.04$ \\
\hline
\end{tabular}

Abbreviations: GL, glycyrrhizin; HPMC, hydroxypropyl methyl cellulose; $\mathrm{Cu} B$, Cucurbitacin B; TPGS, alpha-D-tocopherol polyethylene glycol 1000 succinate.

\section{Redispersibility testing of the granulated powders}

Given the known fact that drying may alter the redispersibility of solid PL-SDC-MMs, a redispersibility study was performed with the successful formulations in terms of coating efficiency. The crude drug powder was used as the control. Figure 2 shows the dissolution curve for the coarse GL and the redispersion profiles for the two granulated powders. Compared with the coarse powder, an obvious increase in dissolution rate can be seen for both granulated powders. However, there was no significant difference in redispersion profiles between the two granulated powders. Furthermore, both of them released completely in 5 minutes. Therefore, we can conclude that, for GL, drying PL-SDC-MMs by spray granulation retains good redispersibility.

Similar results are shown for $\mathrm{Cu}$ B in Figure 3. There is a significant increase in the dissolution rate for the granulated powders compared with coarse $\mathrm{Cu}$ B. No appreciable difference could be found in the redispersion profiles when comparing the two $\mathrm{Cu} \mathrm{B}$ granulated powders except at the first time point, when the formulation containing TPGS was slightly slower. This difference has not been taken into account and has no physical meaning because fast dissolution may result in a fluctuating concentration, and error (of about one minute) in the sampling time may also exist. The difference between this and the GL formulation is that complete redispersion of the two formulations containing $\mathrm{Cu} \mathrm{B}$ takes about 15 minutes. There is no doubt that the redispersibility rate for $\mathrm{Cu} \mathrm{B}$ was reduced by spray granulation. Considering that $\mathrm{Cu} \mathrm{B}$ is completely dissolved in the $\mathrm{Cu} \mathrm{B}-\mathrm{PL} / \mathrm{SDC}$ $\mathrm{MMs}$, the increase in redispersibility time could be due to agglomeration of the nanoparticles as the drug is released. Alternatively, this increase might be caused by the polymer forming the coating that acts as a binder. The polymer needs to swell in water and produce a certain viscosity, which will hinder redispersion of the nanoparticles. However, under comparable conditions, the increase in redispersibility time was not observed with the granulated GL powders, so the latter explanation seems less likely. In order to confirm the former theory, further particle size analysis was carried out.

Particle size analysis for the samples is shown in Figure 4. There are two particle size distribution curves in each figure, ie, one for $\mathrm{Cu}$ B-PL/SDC-MMs without ultrasonication and another for $\mathrm{Cu}$ B-PL/SDC-MMs with ultrasonication. As shown in Figure 4A, ultrasonication had little influence on detection for $\mathrm{Cu}$ B-PL/SDC-MMs. No appreciable difference was found between the two particle size distribution curves. This result is very important for the subsequent study where ultrasonication was used to obtain more information 

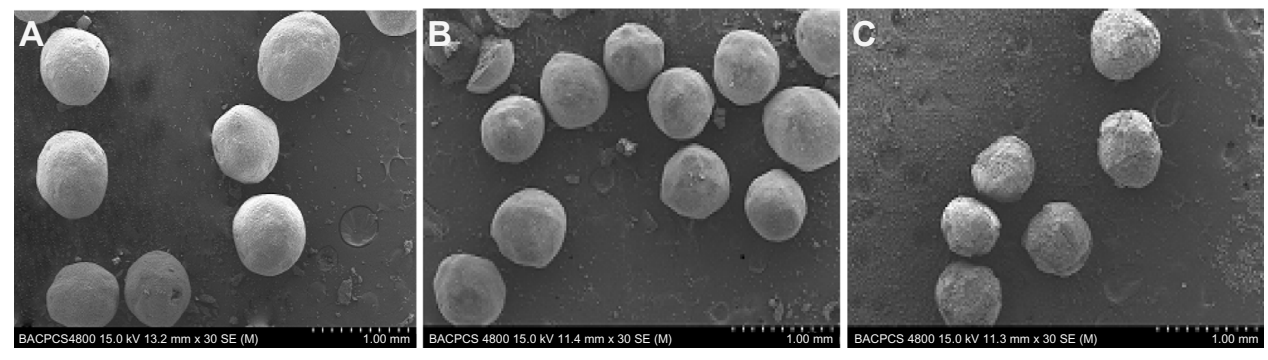

Figure I Scanning electron micrographs of different formulations. (A) Granulated powders containing Cu B-TPGS20-HPMC30, (B) granulated powders containing GLHPMC50, (C) granulated powders containing Cu B-TPGSI0-HPMC30.

Abbreviations: HPMC, hydroxypropyl methyl cellulose; TPGS, alpha-D-tocopherol polyethylene glycol I000 succinate; GL, glycyrrhizin; Cu B, Cucurbitacin B.

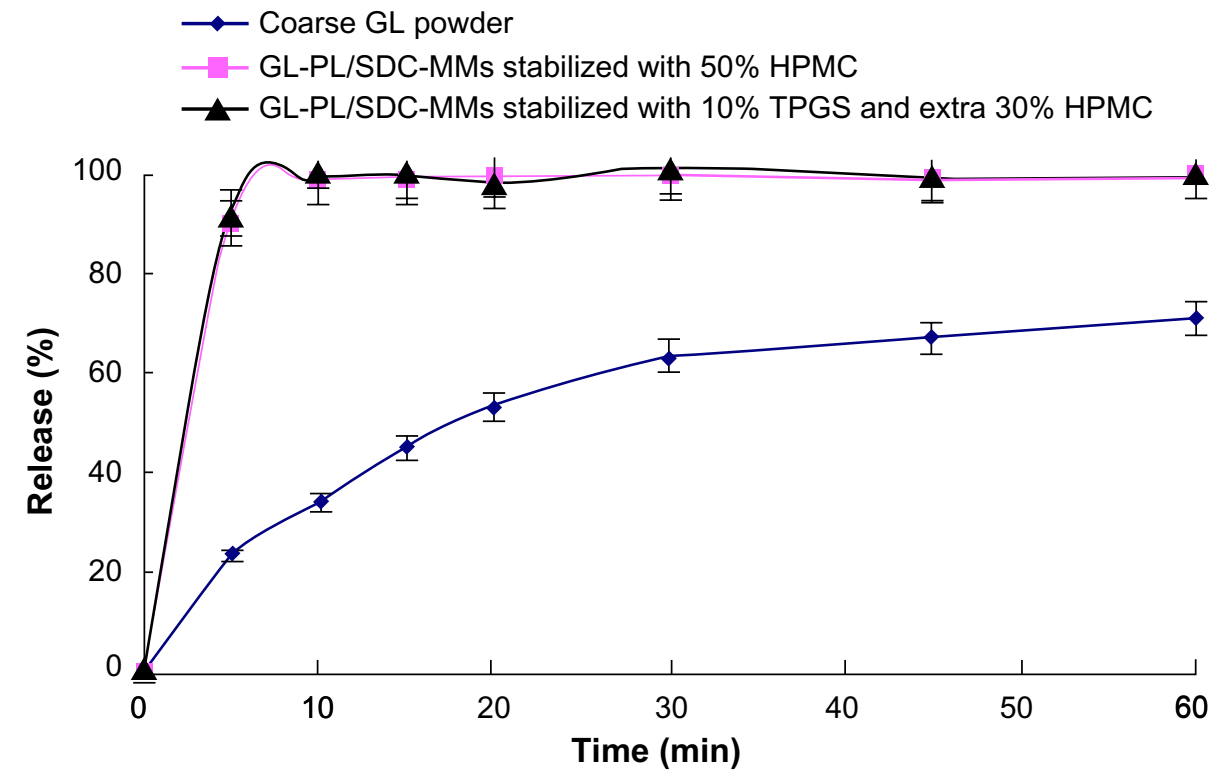

Figure 2 Dissolution curves for $\mathrm{GL}$ in different formulations $(\mathrm{n}=6)$.

Abbreviations: HPMC, hydroxypropyl methyl cellulose; TPGS, alpha-D-tocopherol polyethylene glycol 1000 succinate; SDC, sodium deoxycholate B; GL, glycyrrhizin; PL, phospholipid; MMs, mixed micelles.

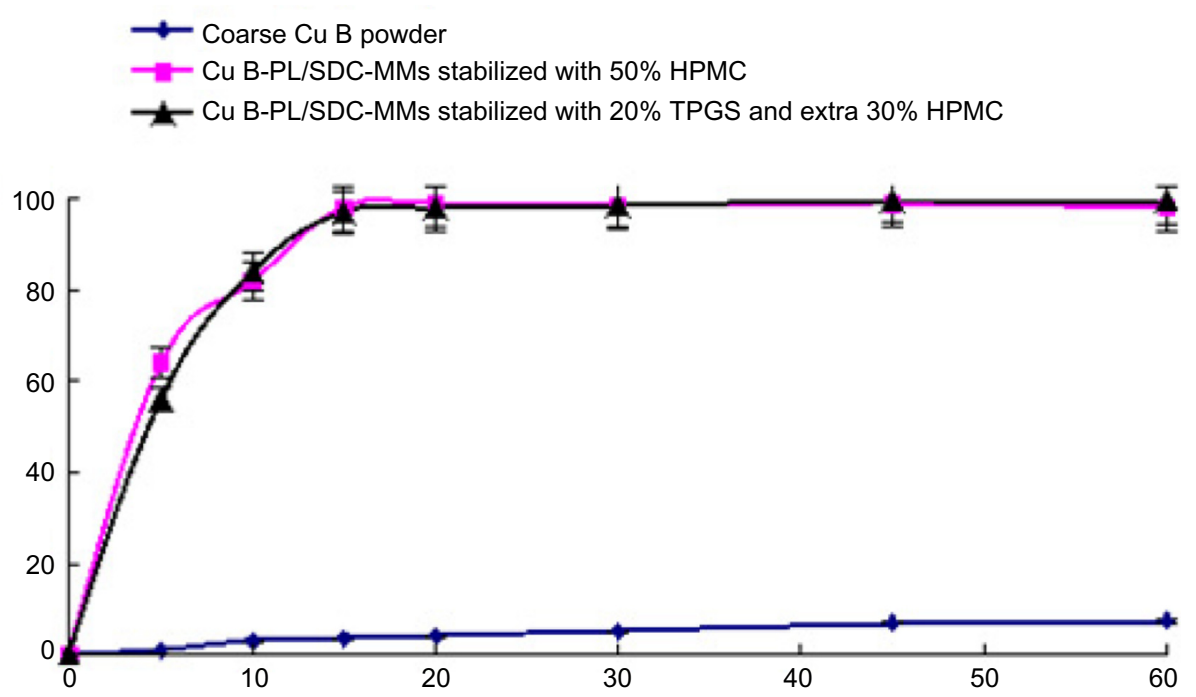

Figure 3 Dissolution curves for $C u B$ in different formulations $(n=6)$.

Abbreviations: HPMC, hydroxypropyl methyl cellulose; TPGS, alpha-D-tocopherol polyethylene glycol 1000 succinate; SDC, sodium deoxycholate B; Cu B, cucurbitacin B; PL, phospholipid; MMs, mixed micelles. 

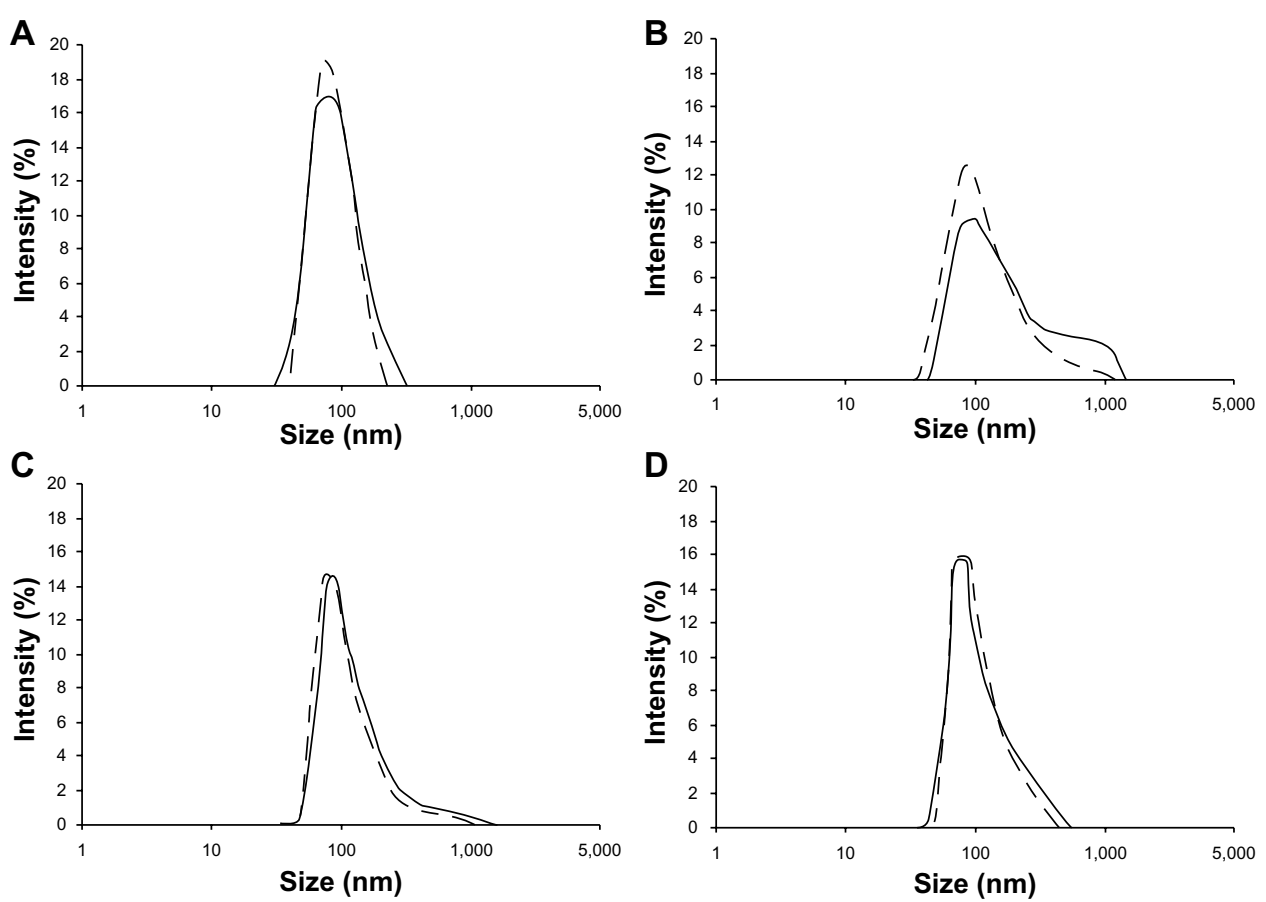

Figure 4 Particle size analysis curves for the granulated powders after release. (A) Curves without ultrasonication (solid line) and with ultrasonication (dotted line) for the Cu B-PL/SDC-MMs. (B) Curves for the Cu B formulation with 50\% HPMC. (C) Curves for the Cu B formulation with $20 \%$ TPGS and $30 \%$ extra HPMC. (D) Curves for the GL formulation with $50 \%$ HPMC.

Abbreviations: HPMC, hydroxypropyl methyl cellulose; SDC, sodium deoxycholate B; GL, glycyrrhizin; Cu B, cucurbitacin B; PL, phospholipid; MMs, mixed micelles; TPGS, alpha-D-tocopherol polyethylene glycol 1000 succinate.

on the granulated powders. The curves for $\mathrm{Cu}$ B-HPMC50 are shown in Figure 4B. The second population in the micrometer range can be clearly observed for the curve measured without ultrasonication. This indicates agglomeration of the nanoparticles. Further, the particle size is decreased (curve shift to the left) by ultrasonication. This also demonstrates the presence of micron particles and suggests breaking up of the agglomerates. Agglomeration also occurred with the $\mathrm{Cu}$ B-TPGS20-HPMC30 granules, but to a much lesser extent, because most of the particles are in the nanometer size range (Figure 4C). This result for the TPGS formulation indicates that TPGS could help to impede agglomeration of nanoparticles. Agglomeration was not observed for the GL formulation (Figure 4D).

\section{In vivo data}

The bioavailability study was undertaken in order to investigate whether the granulated powders maintained good redispersibility in vivo. Different formulations were dosed in fasted male Beagle dogs, ie, the coarse active pharmaceutical ingredient, PL-SDC-MMs, and dried powders of PL-SDC-MMs (with and without TPGS). The pharmacokinetic parameters are summarized in Tables 3 and 4. The plasma concentration versus time curves are shown in Figures 5 and 6 .
The pharmacokinetic parameters and plasma concentration versus time curves for formulations containing GL are shown in Table 3 and Figure 5, respectively. Compared with coarse GL, the area under the curve during 24 hours $\left(\mathrm{AUC}_{0-24}\right)$ for the GL-PL/SDC-MMs, the dried powder containing GLHPMC50, and the dried powder containing GL-TPGS10HPMC30 showed a 2.61-fold, 2.32-fold, and 2.99-fold increase, respectively. The $\mathrm{AUC}_{0-24}$ from all formulations containing the nanoparticles was statistically significant

Table 3 Summary of pharmacokinetic parameters from the in vivo study of $G L$ (mean \pm standard deviation, $n=5$ )

\begin{tabular}{lllll}
\hline Formulation & $\begin{array}{l}\mathbf{C}_{\max } \\
(\mu \mathrm{g} / \mathrm{mL})\end{array}$ & $\begin{array}{l}\mathbf{T}_{\max } \\
(\text { hours })\end{array}$ & $\begin{array}{l}\mathrm{AUC}_{0-24} \\
(\mu \mathrm{g} / \mathrm{mL} / \mathrm{hour})\end{array}$ & $F^{*}$ \\
\hline $\begin{array}{l}\text { GL-PL/SDC-MMs } \\
\text { Dried powder } \\
\text { containing GL- }\end{array}$ & $28.33^{\mathrm{a}} \pm 2.38$ & $0.5 \mathrm{I} \pm 0.1 \mathrm{I}$ & $107.33^{\mathrm{a}} \pm 9.54$ & $2.6 \mathrm{I}$ \\
$\begin{array}{l}\text { HPMC50 } \\
\text { Dried powder }\end{array}$ & $30.12^{\mathrm{a}} \pm 3.87$ & $0.49 \pm 0.13$ & $95.37^{\mathrm{a}} \pm 8.44$ & 2.32 \\
$\begin{array}{l}\text { containing GL- } \\
\text { TPGSI0-HPMC30 }\end{array}$ & & & & \\
Control group & $7.88 \pm 0.94$ & $0.50 \pm 0.08$ & $41.18 \pm 4.92$ & \\
\hline
\end{tabular}

Notes: a $<0.05$ versus control group; *relative bioavailability when compared with control group.

Abbreviations: $\mathrm{AUC}_{0-24}$, area under the curve during 24 hours; GL, glycyrrhizin; PL, phospholipid; HPMC, hydroxypropyl methyl cellulose; TPGS, alpha-D-tocopherol polyethylene glycol 1000 succinate; MMs, mixed micelles; SDC, sodium deoxycholate; $C_{\text {max }}$, peak plasma concentration; $T_{\text {max }}$, time taken to reach peak plasma concentration. 
Table 4 Summary of pharmacokinetic parameters from the in vivo study of $\mathrm{Cu} B$ (mean \pm standard deviation, $n=5$ )

\begin{tabular}{|c|c|c|c|c|}
\hline Formulation & $\begin{array}{l}C_{\max } \\
(\mu g / m L)\end{array}$ & $\mathbf{T}_{\max }(\mathbf{h})$ & $\begin{array}{l}\text { AUC }_{0-24} \\
(\mu \mathrm{g} / \mathrm{mL} / \mathrm{h})\end{array}$ & $F^{*}$ \\
\hline Cu B-PL/SDC-MMs & $9.69^{\mathrm{a}} \pm 1.38$ & $3.01 \pm 0.52$ & $74.94^{\mathrm{a}} \pm 6.03$ & 3.73 \\
\hline $\begin{array}{l}\text { Dried powder } \\
\text { containing } \mathrm{Cu} \\
\text { B-HPMC50 }\end{array}$ & $7.12^{\mathrm{a}-\mathrm{c}} \pm 1.23$ & $3.03 \pm 0.4 I$ & $44.02^{\mathrm{a}, \mathrm{b}} \pm 5.63$ & 2.19 \\
\hline $\begin{array}{l}\text { Dried powder } \\
\text { containing Cu } \\
\text { B-TPGS20-HPMC30 }\end{array}$ & $8.30^{\mathrm{a}, \mathrm{b}} \pm 0.95$ & $3.05 \pm 0.32$ & $49.0 I^{\mathrm{a}, \mathrm{b}} \pm 4.72$ & 2.43 \\
\hline Control group & $3.55 \pm 0.71$ & $3.03 \pm 0.46$ & $20.11 \pm 3.22$ & \\
\hline
\end{tabular}

Notes: ${ }^{a}<0.05$ versus control group; ${ }^{b} P<0.05$ versus $C u$ B-PL/SDC-MMs; $c P<0.05$ compared with dried powder containing Cu B-TPGS20-HPMC30; *relative bioavailability when compared with control group.

Abbreviations: $\mathrm{AUC}_{0-24}$, area under the curve during 24 hours; $\mathrm{Cu} B$, cucurbitacin $\mathrm{B}$ PL, phospholipid; HPMC, hydroxypropyl methyl cellulose;TPGS, alpha-D-tocopherol polyethylene glycol 1000 succinate; MMs, mixed micelles; SDC, sodium deoxycholate; $\mathrm{C}_{\text {max }}$, peak plasma concentration; $\mathrm{T}_{\text {max }}$, time taken to reach peak plasma concentration; h, hours.

$(P<0.05)$ compared with the control group. Compared with the control group $(7.88 \pm 0.94 \mu \mathrm{g} / \mathrm{mL})$, a 3.60-fold, 3.56-fold, and 3.82-fold increase in peak plasma concentration was observed for the GL-PL/SDC-MMs $(28.33 \pm 2.38 \mu \mathrm{g} / \mathrm{mL})$, the dried powder containing GL-HPMC50 $(28.09 \pm 3.11 \mu \mathrm{g} / \mathrm{mL})$, and the dried powder containing GL-TPGS10-HPMC30 $(30.12 \pm 3.87 \mu \mathrm{g} / \mathrm{mL})$, respectively. The difference in values for peak plasma concentration between the control group and the three formulations containing nanoparticles was also statistically significant $(P<0.05)$. However, no statistically significant difference was found between the values for peak plasma concentration and $\mathrm{AUC}_{0-24}$ for the GL-PL/SDC-MMs and its dried counterparts containing GL-HPMC50 and GL-TPGS10-HPMC30. Further, comparable values for time taken to reach peak concentration of $0.51,0.49$, and 0.50 hours were obtained from the GL-PL/SDC-MMs, the dried powder containing GL-HPMC50, and the dried powder containing GL-TPGS10-HPMC30, respectively. These results show that presenting GL in the form of spray granules containing PL-SDC-MMs maintains absorption enhancement as high as that of the liquid PL-SDC-MMs in vivo.

As shown in Table 4 and Figure 6, the values for $\mathrm{AUC}_{0-24}$ and peak plasma concentration obtained for the $\mathrm{Cu}$ B-PL/SDC-MMs, the dried powder containing $\mathrm{Cu}$ B-HPMC50, and the dried powder containing $\mathrm{Cu}$ B-TPGS20HPMC30 were all increased compared with coarse $\mathrm{Cu} B$, and the differences were statistically significant $(P<0.05)$ when compared with the control. However, the values for $\mathrm{AUC}_{0-24}$ and peak plasma concentration for the dried powder containing $\mathrm{Cu}$ B-HPMC50 and the dried powder containing $\mathrm{Cu}$ B-TPGS20-HPMC30 were significantly $(P<0.05)$ lower than the corresponding values for $\mathrm{Cu}$ B-PL/SDC-MMs, indicated that the absorption capability of $\mathrm{Cu} B$ was reduced by spray granulation. This phenomenon might be due to the aggregation of nanoparticles. The peak plasma concentration for the dried powder containing $\mathrm{Cu}$ B-HPMC50 was found to be significantly lower $(P<0.05)$ compared with that of

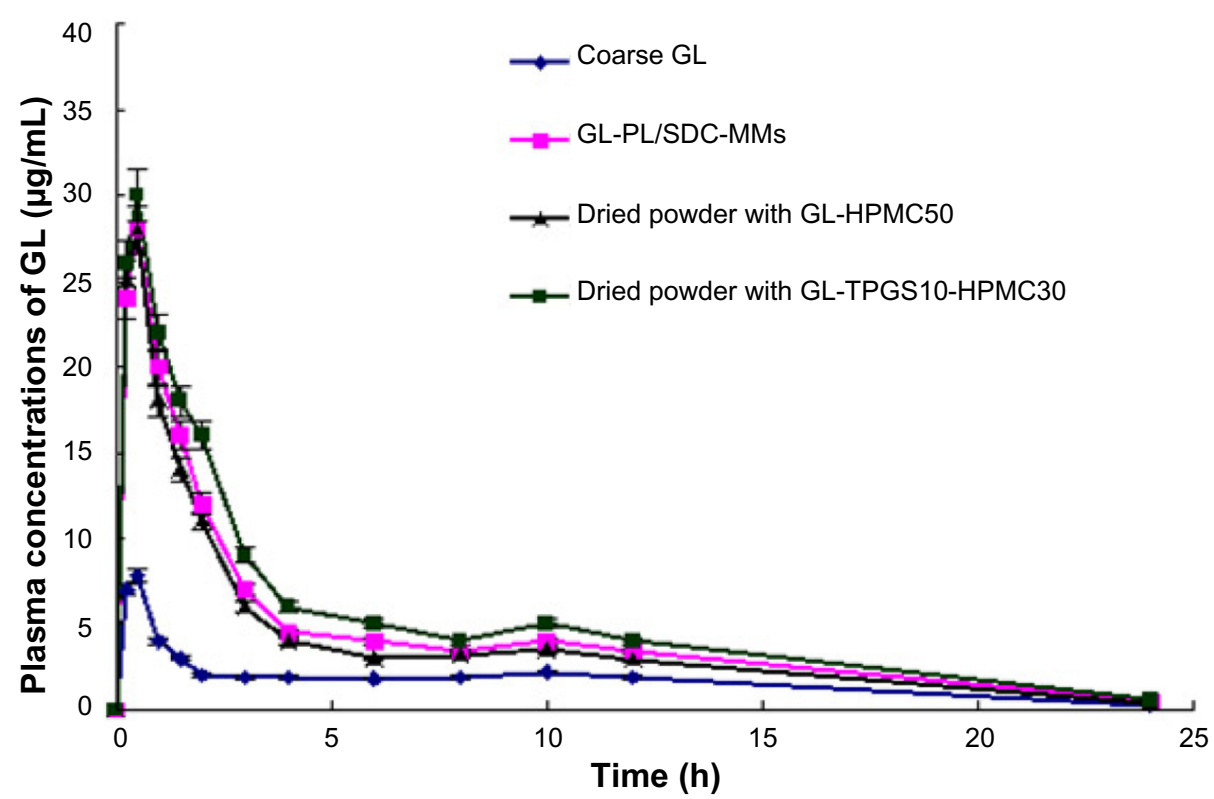

Figure 5 Plasma concentration-time profile for GL in fasted male Beagle dogs after administration of GL in different formulations. Each point represents the mean \pm standard deviation of five experiments.

Abbreviations: HPMC, hydroxypropyl methyl cellulose; SDC, sodium deoxycholate B; GL, glycyrrhizin; PL, phospholipid; MMs, mixed micelles; TPGS, alpha-D-tocopherol polyethylene glycol 1000 succinate; h, hours. 


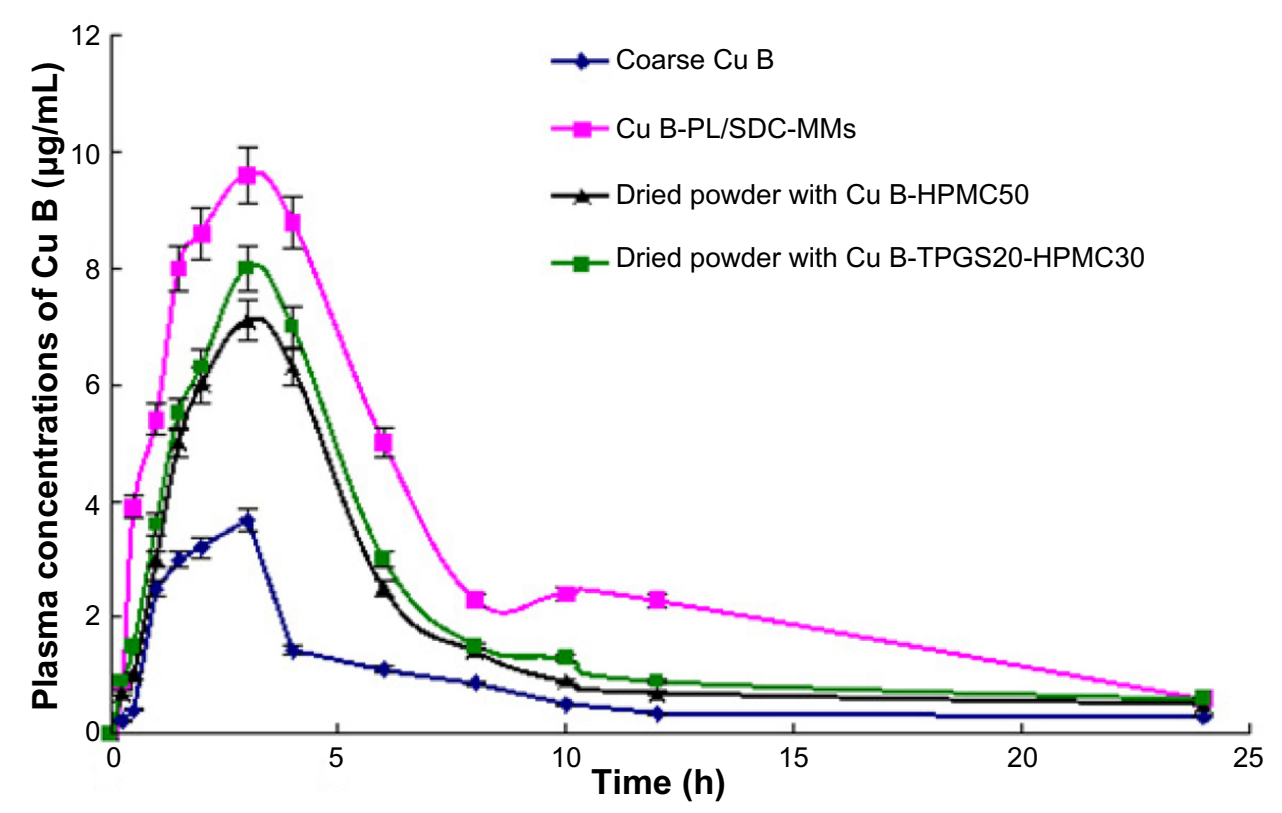

Figure 6 Plasma concentration-time profile for Cu B in fasted male Beagle dogs after administration of Cu B in different formulations. Each point represents the mean \pm standard deviation of five experiments.

Abbreviations: HPMC, hydroxypropyl methyl cellulose; SDC, sodium deoxycholate B; Cu B, Cucurbitacin B; PL, phospholipid; MMs, mixed micelles; TPGS, alpha-Dtocopherol polyethylene glycol 1000 succinate; h, hours.

the dried powder containing $\mathrm{Cu}$ B-TPGS20-HPMC30. The value for the formulation containing TPGS was higher. The explanation for this could be the characteristic nature of TPGS which produces the solubilization effect. This result is different from that for the GL formulations, and a possible explanation could be the different amount of TPGS used in the formulation. The lower amount of TPGS used in the dried powder containing GL-TPGS10-HPMC30 was not adequate to produce the solubilization effect.

These in vitro and in vivo data demonstrate that the hydrophobicity of a drug has an influence on its redispersion profile after drying by spray granulation. $\mathrm{Cu} \mathrm{B}$, which was more difficult to form into PL-SDC-MMs due to its greater hydrophobicity compared with GL, was more difficult to prepare into a spray granulation formulation with a good re-dispersion profile. This study indicates that the ability to generate stable PL-SDC-MMs does not necessarily result in favorable spray granulation formulations with fast redispersibility and no agglomeration. Regardless of the drying technique used, the hydrophobicity of a compound will definitely affect the quality of a product after drying.

\section{Conclusion}

In this study, the PL-SDC-MMs were dried by spray granulation and several parameters were identified to have a significant influence on the result of the drying process. The right type and amount of stabilizer or protectant plays an important role in adhering nanoparticles to a substrate. Our study also suggests that successful spray granulation (the coating efficiency was equal to or more than 90\%) will not necessarily result in a favorable nanoparticle redispersion profile since the quality of a product after drying is closely related to the hydrophobicity of drug. Good results were obtained for GL, with complete redispersion within 5 minutes in vitro and improved absorption as high as liquid PL-SDC-MMs in vivo. However, for $\mathrm{Cu} \mathrm{B}$, complete redispersion took place within approximately 15 minutes in vitro and the dried powders could not maintain good redispersibility in vivo. The explanation for the difference between $\mathrm{Cu} \mathrm{B}$ and GL could be the aggregation of $\mathrm{Cu} \mathrm{B}$ nanoparticles due to its higher hydrophobicity. This study confirms that successful preparation of stable PL/SDC-MMs cannot be guaranteed to produce a successful spray granulation formulation.

\section{Acknowledgment}

This study was supported by the National Key New Drugs Innovation Foundation (2013ZX09J13109-06C), the Natural Science Foundation of Beijing City of China (7122176), and the Scientific Research Foundation for Returned Overseas Chinese Scholars, State Education Ministry (20101561).

\section{Disclosure}

The authors report no conflicts of interest in this work. 


\section{References}

1. Desai PP, Date AA, Patravale VB. Overcoming poor oral bioavailability using nanoparticle formulations - opportunities and limitations. Drug Discov Today. 2011;9:e87-e95.

2. Lipinski CA. Drug-like properties and the causes of poor solubility and poor permeability. J Pharmacol Toxicol Methods. 2000;44:235-249.

3. Lipinski CA. Poor aqueous solubility - an industry wide problem in drug discovery. Am Pharm Rev. 2002;5:82-85.

4. Lei Y, Lu Y, Qi J, et al. Solid self-nanoemulsifying cyclosporin A pellets prepared by fluid-bed coating: preparation, characterization and in vitro redispersibility. Int J Nanomedicine. 2011;6:795-805.

5. Teelmann K, Schläppi B, Schüpbach M, Kistler A. Preclinical safety evaluation of intravenously administered mixed micelles. Arzneimittelforschung. 1984;34:1517-1523.

6. Hammad MA, Müller BW. Solubility and stability of tetrazepam in mixed micelles. Eur J Pharm Sci. 1998;7:49-55.

7. Dong F, Xie Y, Qi J, et al. Bile salt/phospholipid mixed micelle precursor pellets prepared by fluid-bed coating. Int J Nanomedicine. 2013;8: 1653-1663.

8. Duan RL, Sun X, Liu J, et al. Mixed micelles loaded with silybin-polyene phosphatidylcholine complex improve drug solubility. Acta Pharmacol Sin. 2011;32:108-115.

9. Yu JN, Zhu Y, Wang L, et al. Enhancement of oral bioavailability of the poorly water-soluble drug silybin by sodium cholate/phospholipidmixed micelles. Acta Pharmacol Sin. 2010;31(6):759-764.

10. Jin S, Fu S, Han J, et al. Improvement of oral bioavailability of glycyrrhizin by sodium deoxycholate/phospholipid-mixed nanomicelles. J Drug Target. 2012;20:615-622.

11. Lv QY, Shen CY, Li XY, et al. Mucoadhesive buccal films containing phospholipid-bile salts-mixed micelles as an effective carrier for Cucurbitacin B delivery. Drug Delivery. In press 2013.

12. Peters RR, Saleh TF, Lora M, et al. Anti-inflammatory effects of the products from Wilbrandia ebracteata on carrageenan-induced pleurisy in mice. Life Sci. 1999;64:2429-2437.

13. Yesilada E, Tanaka S, Sezik E, Tabata M. Isolation of an antiinflammatory principle from the fruit juice of Ecballium elaterium. J Nat Prod. 1988;51:504-508.
14. Agil A, Miró M, Jimenez J, et al. Isolation of ani-hepatotoxic principle form the juice of Ecballium elaterium. Planta Med. 1999;65: 673-675.

15. Hou YC, Hsiu SL, Ching H, et al. Profound difference of metabolic pharmacokinetics between pure glycyrrhizin and glycyrrhizin in licorice decoction. Life Sci. 2005;76:1167-1176.

16. Okimasu E, Moromizato Y, Watanabe S, et al. Inhibition of phospholipase A2 and platelet aggregation by glycyrrhizin, an antiinflammation drug. Acta Med Okayama. 1983;37:385-391.

17. Nagai T, Egashira T, Kudo Y, et al. Attenuation of dysfunction in the ischemia-reperfused liver by glycyrrhizin. Jpn J Pharmacol. 1992;58: 209-218.

18. Komatsu H, Kitajima A, Okada S. Pharmaceutical characterization of commercially available intravenous fat emulsions: estimation of average particle size, size distribution and surface potential using photon correlation spectroscopy. Chem Pharm Bull. 1995;43:1412-1415.

19. Müller RH, Mäder K, Gohla S. Solid lipid nanoparticles (SLN) for controlled drug delivery-a review of the state of the art. Eur J Pharm Biopharm. 2000;50:161-177.

20. Müller RH, Rühl D, Runge SA. Biodegradation of solid lipid nanoparticles as a function of lipase incubation time. Int J Pharm. 1996;144: 115-121.

21. Liu J, Gong T, Wang CG, et al. Solid lipid nanoparticles loaded with insulin by sodium cholate-phosphatidylcholine-based mixed micelles: preparation and characterization. Int J Pharm. 2007;340:153-162.

22. Bose S, Schenck D, Ghosh I, et al. Application of spray granulation for conversion of a nanosuspension into a dry powder form. Eur J Pharm Sci. 2012;47:35-43.

23. Bouffard J, Kaster M, Dumont, H. Influence of process variables and physico-chemical properties on the granulation mechanism of mannitol in a fluid bed top spray granulator. Drug Dev Ind Pharm. 2005;31: 923-933.

24. Kayaert P, Anné M, Van den Mooter G. Bead layering as a process to stabilize nanosuspensions: influence of drug hydrophobicity on nanocrystal reagglomeration following in-vitro release from sugar beads. J Pharm Pharmacol. 2011;63:1446-1453.
International Journal of Nanomedicine

\section{Publish your work in this journal}

The International Journal of Nanomedicine is an international, peerreviewed journal focusing on the application of nanotechnology in diagnostics, therapeutics, and drug delivery systems throughout the biomedical field. This journal is indexed on PubMed Central,

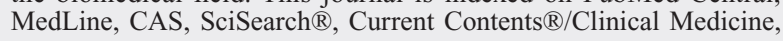

\section{Dovepress}

Journal Citation Reports/Science Edition, EMBase, Scopus and the Elsevier Bibliographic databases. The manuscript management system is completely online and includes a very quick and fair peer-review system, which is all easy to use. Visit http://www.dovepress.com/ testimonials.php to read real quotes from published authors. 